\title{
Erratum to: On Completely Regular Codes
}

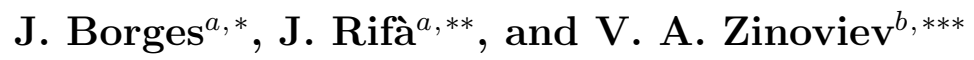 \\ ${ }^{a}$ Department of Information and Communications Engineering, \\ School of Engineering, Universitat Autonoma de Barcelona, Bellaterra, Spain \\ ${ }^{b}$ Kharkevich Institute for Information Transmission Problems, \\ Russian Academy of Sciences, Moscow, Russia \\ e-mail: *joaquim.borges@uab.cat, ${ }^{* *}$ josep.rifa@uab.cat, ${ }^{* * *}$ zinov@iitp.ru
}

Received April 30, 2019; revised April 30, 2019; accepted May 21, 2019

\begin{abstract}
We correct mistakes in the formulations of Theorem 19 and Proposition 17 of the original article, published in vol. 55, no. 1, 1-45.
\end{abstract}

DOI: $10.1134 / \mathrm{S} 0032946019030098$

In the original article, the authors made the following mistakes in formulations.

1. Theorem 19 should read as follows:

Theorem 19 [60,61]. If $C$ is a perfect nontrivial e-code in the Johnson scheme $J(n, w)$, $w \leq n / 2$, then its length $n$ is upper bounded as follows:

$$
n \leq \frac{2 e+1}{e}(w-1) .
$$

2. Proposition 17 should read as follows:

Proposition 17. If $C$ is a nontrivial perfect e-code in $J(n, w)$, then $w$ is lower bounded as follows:

$$
w \geq \begin{cases}e^{2}+3 e+1 & \text { if } w>n / 2[61], \\ \frac{1}{2} e(e+1)(e+2)+2 e+2 & \text { if } w<n / 2 \text { and } n \text { odd [53], } \\ e(e+1)(e+2)+2 e+2 & \text { if } w<n / 2 \text { and } n \text { even [53], } \\ 2 e^{2}+4 e+1 & \text { if } w=n / 2[53] .\end{cases}
$$

The authors apologize for the mistakes.

The original article can be found online at https://doi.org/10.1134/S0032946019010010. 